\title{
Implementation and adoption of advanced care planning in the elderly trauma patient

\author{
K. Verhoeff ${ }^{1}$, P. Glen², A. Taheri' ${ }^{2}$ B. Min ${ }^{1}$, B. Tsang ${ }^{2}$, V. Fawcett ${ }^{2}$ and S. Widder ${ }^{2 *}$
}

\begin{abstract}
Background: Geriatric trauma has high morbidity and mortality, often requiring extensive hospital stays and interventions. The number of geriatric trauma patients is also increasing significantly and accounts for a large proportion of trauma care. Specific geriatric trauma protocols exist to improve care for this complex patient population, who often have various comorbidities, pre-existing medications, and extensive injury within a trauma perspective. These guidelines for geriatric trauma care often suggest early advanced care planning (ACP) discussions and documentation to guide patient and family-centered care.

Methods: A provincial ACP program was implemented in April of 2012, which has since been used by our level 1 trauma center. We applied a before and after study design to assess the documentation of goals of care in elderly trauma patients following implementation of the standardized provincial ACP tool on April 1, 2012.

Results: Documentation of ACP in elderly major trauma patients following the implementation of this tool increased significantly from 16 to 35\%. Additionally, secondary outcomes demonstrated that many more patients received goals of care documentation within $24 \mathrm{~h}$ of admission, and $93 \%$ of patients had goals of care documented prior to intensive care unit (ICU) admission. The number of trauma patients that were admitted to the ICU also decreased from 17 to $5 \%$.

Conclusion: Early advanced care planning is crucial for geriatric trauma patients to improve patient and family-centered care. Here, we have outlined our approach with modest improvements in goals of care documentation for our geriatric population at a level 1 trauma center. We also outline the benefits and drawbacks of this approach and identify the areas for improvement to support improved patient-centered care for the injured geriatric patient. Here, we have provided a framework for others to implement and further develop.
\end{abstract}

Keywords: Quality improvement, Geriatric trauma, Advanced care planning, Patient and family-centered care

\section{Background}

The number of Canadian seniors is increasing significantly, with people over the age of 65 expected to compose $25 \%$ of the country's population by 2036 [1]. A proportional increase in geriatric trauma is expected [2,3]. Geriatric trauma is associated with longer intensive care unit (ICU) admissions, more life-threatening complications while in hospital, and significantly higher mortality despite similar injury severity when compared to younger patients [2-6].

The American College of Surgeons Committee on Trauma (ACS COT) has defined specific protocols for geriatric trauma in order to improve management of

\footnotetext{
* Correspondence: Sandy.Widder2@albertahealthservices.ca

${ }^{2}$ Department of Surgery, 2D4.27 Mackenzie Health Sciences Centre,

University of Alberta, 8440-112 St. Edmonton, Edmonton, Alberta T6G 2B7,

Canada

Full list of author information is available at the end of the article
}

comorbid conditions, polypharmacy, poor physiologic reserve, and elderly specific complications (i.e., delirium) [2]. ACS COT suggests involving the patient, surrogate decision-makers, family, and the health care team in a discussion about the patient's treatment priorities. This conversation aims to establish and document the commonly termed "goals of care" (GOC) within the first $72 \mathrm{~h}$ after admission and is meant to weigh the benefits of escalating treatments against the risks of complications [2].

Despite trauma being a leading cause of death in Canada, there is a relative dearth of studies focusing on GOC discussions in trauma patients [7]. Advanced care planning (ACP) facilitates discussions around patient's health care goals (i.e., cardiopulmonary resuscitation) along with a realistic presentation of what intensive, 
invasive treatments can provide. ACP processes can guide complex treatment decision-making (including end-of-life discussions between allied health professionals, patients, and their families) to promote patient and family-centered care (PFCC) and eliminate uncertainty regarding the patient's wishes $[8,9]$.

The province of Alberta implemented an ACP tool in April of 2012, including within our level 1 trauma center. The program involved providing every patient and family with information sheets regarding ACP, prompting a discussion about future care between the patient and care providers regarding the patient's care goals, and ultimately documenting these goals of care in the chart (see Additional file 1). A specific GOC order sheet was also provided to practitioners for process standardization (see Additional file 1). We sought to determine the effect of this ACP tool on establishing documented GOC for geriatric trauma patients. We also aimed to outline the benefits and drawbacks of this approach and identify the areas for improvement to assist others who wish to implement an ACP system within their trauma systems.

\section{Methods}

We applied a before and after study design to assess the documentation of GOC in elderly trauma patients following implementation of the standardized provincial ACP tool on April 1, 2012 (see Additional file 1). The Alberta Trauma Registry is a prospective database that collects information on all major trauma patients (Injury Severity Score $\geq 12$ ). This database was retrospectively queried for a cohort of patients over the age of 65 who were admitted from April 1, 2010, to September 30, 2014, representing a period of 24 months before and 30 months after ACP implementation. Charts for this cohort were individually reviewed to determine patient demographics, whether GOC were documented, whether it was done within $24 \mathrm{~h}$ of admission and prior to ICU admission, the source of the GOC information, and the patient's post-hospitalization disposition.

Data was separated into a pre-intervention group $(n=177)$ that included patients 24 months prior to April 2012 and a post-intervention group $(n=294)$ that included patients 30 months after ACP program implementation. A 2-year before and after time frame was chosen to adequately assess for change following the project implementation. As the ACP tool was implemented provincially, an additional 6 months was included within the analysis for the after group to allow for a period of institutional uptake of these processes. The groups were compared based on Injury Severity Score (ISS), sex, age, length of stay, and mechanism of injury, using chi-squared tests of independence. The primary outcome measure of this study was whether the ACP intervention was associated with a change in the documentation of GOC in the chart overall; a comparison between both time periods was made using Fisher's exact test. Secondary measures assessed documentation of GOC within $24 \mathrm{~h}$ and upon admission to ICU. These were also assessed with Fisher's exact test.

\section{Results}

The two groups of geriatric trauma patients in this study were similar with regard to age, sex, mechanism of injury, length of stay, ISS, and disposition after discharge (Table 1). Patients were approximately 77 years old in both groups with 35\% of patients before 2012 and $40 \%$ after 2012 being female (Table 1). Importantly, the mean ISS for patients in both groups was statistically similar, being 23 before the ACS program and 22 after. Patients primarily suffered blunt traumatic injuries from either falls or motor vehicle accidents, a distribution that did not change between study periods (Table 1 ).

Analysis of the primary outcome demonstrated a statistically significant change $(p<0.01)$ in GOC documentation following the ACP program implementation. Twenty-four months prior to the ACP program implementation, only $16 \%$ of major geriatric trauma

Table 1 Patient demographics

\begin{tabular}{|c|c|c|c|}
\hline & Before $(n=177)$ & After $(n=294)$ & p value \\
\hline Mean age (years) & 76.50 & 77.02 & 0.51 \\
\hline Mean ISS & 22.65 & 22.13 & 0.50 \\
\hline Median LOS (days) & 9 & 7 & $\mathrm{~N} / \mathrm{A}$ \\
\hline Number female (proportion) & $60(0.34)$ & $117(0.40)$ & 0.92 \\
\hline Number male (proportion) & $117(0.66)$ & $177(0.60)$ & 0.94 \\
\hline Injury & $n(\%)$ & $n(\%)$ & \\
\hline Blunt & $174(98)$ & $289(98)$ & 0.99 \\
\hline Fall & $118(68)$ & $207(72)$ & 0.96 \\
\hline Motor vehicle & $35(20)$ & $57(20)$ & 0.99 \\
\hline Pedestrian & $8(5)$ & $9(3)$ & 0.94 \\
\hline Other & $13(7)$ & $16(6)$ & 0.94 \\
\hline Penetrating & $0(0)$ & $1(0)$ & 0.94 \\
\hline Burn & $3(2)$ & $4(1)$ & 0.98 \\
\hline Other & $0(0)$ & $1(0)$ & 0.94 \\
\hline \multicolumn{4}{|l|}{ Disposition } \\
\hline Home & $73(41)$ & $127(43)$ & 0.97 \\
\hline Home support & $13(7)$ & $10(3)$ & 0.84 \\
\hline Other hospital & $35(20)$ & $49(17)$ & 0.94 \\
\hline Rehabilitation & $13(7)$ & $30(10)$ & 0.92 \\
\hline Chronic care & $20(11)$ & $27(9)$ & 0.95 \\
\hline Nursing home & $7(4)$ & $4(1)$ & 0.83 \\
\hline Died & $16(9)$ & $47(16)$ & 0.84 \\
\hline
\end{tabular}

Characteristics of geriatric trauma population before and after the implementation of the advanced care planning project at our hospital 
patients had documented GOC by the end of their hospital stay (Table 2). This improved to $35 \%$ in the study period following ACP project implementation.

Secondary outcomes demonstrated that GOC documentation within $24 \mathrm{~h}$ was significantly higher after the intervention (Table 2). For the 30 months following April 2012, 21\% of geriatric major trauma patients had this documentation done within $24 \mathrm{~h}$ as compared to $11 \%$ in the 24 months prior to ACP implementation.

The rate of admission to the ICU was significantly lower after the ACP program implementation when compared to before (Table 3). There was also a significant increase in the documentation of GOC for patients admitted to the ICU with $93 \%$ of admitted ICU patients having this documented after the ACP implementation, as compared to $20 \%$ prior to this project.

\section{Discussion}

Despite the importance of ACP, a 2008 report from the US Congress demonstrated the widespread failure of implementation [10]. Some publications have advocated to abandon advanced care directives, citing reasons such as patient uncertainty about their use, inadequate prognostication to guide discussions, and poor compliance by health care practitioners with patient wishes [11-13]. However, when achieved ACP has been shown to significantly reduce anxiety, depression, and posttraumatic stress disorder in the relatives of patients who die $[8,14,15]$. Even when ACP was done but not enacted, patient and family satisfaction with their care was significantly improved [14]. This correlation addresses the vital importance of patient autonomy and PFCC to guide treatment decisions. It is also vitally important to separate the notion of ACP and end of life care decision-making. The two are independent, with the ACP discussion establishing patient care priorities.

Table 2 Successful goals of care documentation

\begin{tabular}{llll}
\hline Goals of care & $\begin{array}{l}\text { Before }(n=177) \\
n(\%)\end{array}$ & $\begin{array}{l}\text { After }(n=294) \\
n(\%)\end{array}$ & $p$ value \\
\hline $\begin{array}{llll}\text { Documented } \\
\text { Yes }\end{array}$ & $28(16)$ & $103(35)$ & \\
No & $149(84)$ & $191(65)$ & $<0.01$ \\
Within 24h & & & \\
$\quad$ Yes & $20(11)$ & $61(21)$ & \\
No & $157(89)$ & $233(79)$ & $<0.01$ \\
Source & & & \\
$\quad$ Patient & $1(4)$ & $2(2)$ & \\
$\quad$ Substitute decision-maker & $26(93)$ & $87(84)$ & \\
Advance directive & $1(4)$ & $14(14)$ & ns \\
\hline
\end{tabular}

Documentation of goals of care before and after the implementation of the advanced care planning project at our hospital
Table 3 Admission to ICU and rate of GOC documentation

\begin{tabular}{llll}
\hline & $\begin{array}{l}\text { Before }(n=177) \\
n(\%)\end{array}$ & $\begin{array}{l}\text { After }(n=294) \\
n(\%)\end{array}$ & $p$ value \\
\hline Admitted to ICU & $30(17)$ & $14(5)$ & $<0.01$ \\
$\begin{array}{l}\text { Goals documented } \\
\text { Yes }\end{array}$ & $6(20)$ & $13(93)$ & \\
No & $24(80)$ & $1(7)$ & $<0.01$ \\
\hline
\end{tabular}

Documentation of goals of care before and after the implementation of the advanced care planning project for patients admitted to the intensive care unit (ICU)

If the patient's priorities do not fit with achievable improvements in their condition, then end of life care discussions may be required. However, the initial discussion should focus on establishing priorities in care. We outline our institution's improvements for geriatric trauma patients following the implementation of a provincial ACP tool and discuss the areas for potential improvement.

As the management of traumatic injuries improves and patient expectations of autonomy are reinforced, the practice of ACP will become increasingly important [3-6]. Prior to the implementation of Alberta's provincial ACP program, only $16 \%$ of our level one trauma center's major trauma patients over 65 years old had documented GOC by the end of their hospital stay (Table 2). Following the implementation of a provincial ACP program, GOC documentation changed significantly, with an increase to $34 \%$ (Table 2). Although we achieved improvement, this result shows that many patients did not have their goals documented-indicating that barriers continue to exist that limit ACP discussions. The source of the GOC decision was documented in our study and shows that in both the before and after groups, most decisions regarding GOC were provided by a substitute decision-maker or an advanced care directive (Table 2). This is likely because geriatric trauma patients have injuries, co-morbidities, and complications (i.e., delirium) that would prevent them from making GOC decisions. This also highlights the importance of care conferences that focus on PFCC early during geriatric trauma admission to support $\mathrm{GOC}$ decisions.

Due to the high risk of intervention, prolonged ICU admission, life-threatening complications, and mortality in geriatric trauma, early ACP discussion is key $[2,4-6]$. Following implementation of our ACP tool, new ACS COT guidelines suggested ACP and documentation of GOC within $72 \mathrm{~h}$ of admission for geriatric trauma patients [2]. Secondary outcomes to evaluate GOC documentation in the first $24 \mathrm{~h}$ and prior to ICU admission were chosen as two identifiable time points when geriatric patients were likely to undergo interventions. However, if ACS COT guidelines were released prior to study implementation, a 72-h period may have been chosen to assess our compliance with these guidelines. Despite that, our ACP program implementation was associated 
with increased early GOC documentation within the first $24 \mathrm{~h}$ (Table 2).

A substantial increase in GOC prior to ICU admission is also noted from 20 to $93 \%$ with an associated decrease in ICU admission from 17 to 5\% (Table 3). There was an obvious culture shift within the ICU to initiate GOC documentation on transfer to their unit, which may have even prevented undesired transfers to the ICU, whereby risks of intensive invasive care would have outweighed benefits to the patient. We suggest that ICU providers are likely accustomed to undergoing ACP discussion due to their highly acute patient population and specialized training around PFCC and communication, lending to the ease of transition for their unit. It may be beneficial to involve these types of practitioners in GOC discussion early in a geriatric trauma patient's hospital admission. Having these practitioners involved with medical education to provide tools and approaches for these discussions may also be beneficial.

Of note, there was a statistically insignificant mortality increase after ACP project implementation (Table 1). This may relate to fewer patients desiring ICU care and heroic lifesaving measures. There was also an accompanying statistically insignificant trend towards fewer patients sent to chronic care or a nursing home following their trauma admission. However, these results should be evaluated cautiously, as our study lacked the power to isolate the significance of these trends.

Successful programs such as "Respecting Choices," "Physician Orders for Life-Sustaining Treatment," and others outlined important tools for ACP implementation that our provincial program modeled [14, 16]. These included a need for leaders to enact change, implementation in a medical culture receptive to change, and complex ACP that focused on discussions rather than specific interventions $[14,16]$. Ensuring ACP and documentation of GOC prior to deterioration in condition is vitally important to ensure escalated care can be accessed expediently, when appropriate $[10,16]$. The ACP program also increased GOC documentation in the first $24 \mathrm{~h}$ (Table 2), which is important for geriatric trauma patients who often require rapid interventions soon after admission. A final important aspect of successful ACP project implementation that we modeled is an openness to department-specific quality improvement, as ACP is diverse and can take many forms even within a single hospital [14-16]. This will allow our project to continue building on its success in the future within our own institution as we seek opportunities to educate our physicians on the importance of having ACP discussions.

Despite ACP program implementation and a significant change in GOC documentation, we are seeking ways to increase the documentation beyond the $35 \%$ level we have achieved in our current study. Achievable, identifiable, and clinically significant pathways to implementing effective ACP have been demonstrated, and we continue to follow these models to further improve our ACP practices $[8,14-16]$. The barriers to ACP in these studies include staff availability and time, their ability to confidently and accurately discuss ACP, and organizational commitment and support for ACP programs [14, 17-19]. Similarly, focusing on specific advanced directives, rather than a complex and complete understanding of the patient's GOC, leads to non-documented and ineffective ACP $[13,14,19,20]$.

\section{Lessons and limitations}

Future initiatives that may assist our trauma services division to improve ACP for geriatric trauma patients exist. Ensuring ACP prior to emergency care, such as by primary care providers in family practice-so-called advance directives-has been used by other programs to improve ACP and reduce time constraints for acute care providers in the emergency setting $[10,16]$. It is important to recognize that while $\mathrm{ACP}$ was increased through this project, $93 \%$ of GOC decisions in the before group and $84 \%$ in the after group were made via a substitute decision maker; this highlights the importance of discussing ACP while patients are healthy to maintain their autonomy in acute scenarios. Initiating these family discussions and decisions should be standard of care for all patients that are admitted to hospital and should be encouraged in the outpatient population as well. Emphasizing the importance of ACP during trauma educational initiatives and outreach programs within the community may improve pre-trauma GOC documentation or discussion; as mentioned, involving experienced intensivists to assist education may provide insight into tools and approaches for these difficult discussions.

Time constraint concerns both in the primary care and emergency care settings were identified by practitioners early in the development of model ACP programs and may be an area for future examination [16]. While the resources do not exist to employ individuals solely for the purposes of ACP discussion, the provincial program suggested that all health care practitioners (including nurses, social workers, respiratory therapists, spiritual health practitioners, and all allied health professionals) play a role in ACP by following five "Core Elements of ACP" (Fig. 1) [16]. Involving specialists, such as intensivists, geriatricians, and palliative care specialists, early in the care of geriatric trauma patients is likely beneficial, especially if a shared mental model between the care team and patient and family cannot be achieved. In order to implement these elements in our program and limit time constraint issues, the culture and approach to ACP will likely require champions for change 


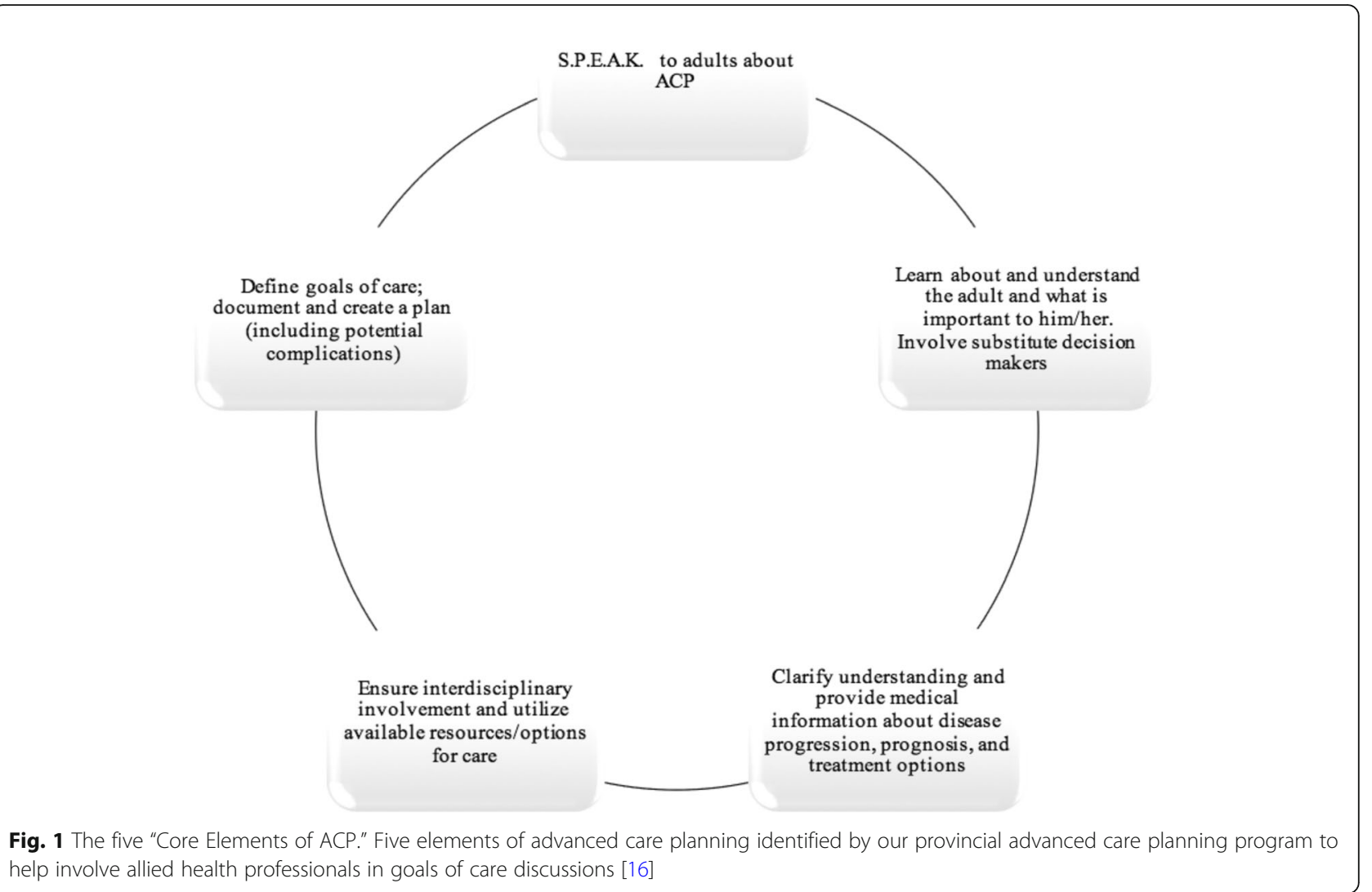

to lead the way, as other successful programs have demonstrated $[14,16]$.

Despite demonstrating improved GOC documentation, the authors recognize certain shortcomings of this method for evaluating ACP program implementation. A before and after study design cannot exclude that other changes or that baseline improvement led to the results we discovered. An interrupted time series analysis to describe the baseline increase in GOC documentation could help attribute the results of our study more directly to the intervention. Also, our comparison groups would ideally be similar in size; however, this is likely accounted for by increasing geriatric trauma volume at our center and the 6-month transition period in our post-intervention group. Our study also associated GOC documentation with ACP discussions; however, we did not evaluate the comprehensiveness of the ACP discussions, or whether they led to a change in intervention and procedures for geriatric trauma patients. Additional studies would be needed to evaluate if the change in GOC documentation and ACP over the study period altered or improved care. Our study attempted to assess mortality and disposition but did not possess sufficient power to determine any change. Future studies may endeavor to include these changes and assess other clinical outcomes to better evaluate the implementation of their ACP programs.

\section{Conclusion}

The provincial advanced care planning program applied to major geriatric trauma patients at our hospital successfully increased GOC documentation from 16 to $35 \%$. Additionally, $21 \%$ of patients received had documentation within the first $24 \mathrm{~h}$ of their admission versus $11 \%$ prior to the project. This demonstrates that ACP programs can improve GOC documentation within the geriatric trauma environment. Additional quality improvement is required to further increase ACP use at our hospital; however, many other programs have shown successful ACP implementation in similar patients and provide various areas for improvement [14-16]. We posit that the most significant factors for ACP improvement using our program will involve additional educational support on the importance of these discussions, the involvement of specialists' adept at these discussions, and leaders to promote effective culture change about ACP at our hospital. While this process can be slow, as demonstrated by others, effective ACP is required for proper patientcentered care for the growing geriatric trauma patient population. 


\section{Additional file}

Additional file 1: Advanced care planning bedside tool and order set Pdf. file that outlines the advanced care planning "goals of care" order set and a document that is provided to patients and their families to assist with decision making and thoughtful advanced care planning. (PDF 2053 kb)

\section{Abbreviations}

ACP: Advanced care planning; ACS COT: American College of Surgeons Committee on Trauma; GOC: Goals of care; ICU: Intensive care unit; ISS: Injury Severity Score; PFCC: Patient and family-centered care

\section{Acknowledgements}

The authors wish to thank the staff from the Health Records at the University of Alberta Hospital for their assistance and effort towards the collection of patient charts for this project.

\section{Funding}

This work was not supported by any funding, and no funding body was involved with the design of the study; collection, analysis, and interpretation of data; or with any writing for this manuscript.

\section{Availability of data and materials}

The datasets used and/or analyzed during the current study are available from the corresponding author on reasonable request.

\section{Authors' contributions}

KV collected the patient data, drafted the manuscript, and assisted with the editing. PG assessed the data, drafted the manuscript, and assisted with the editing. AT assisted with the data assessment and editing. BM collected the patient data and assisted with the drafting of the manuscript. BT and VF assisted with the project strategy and edited the manuscript. SW initiated the project implementation and strategy and edited the manuscript. All authors have read and approved the final manuscript.

\section{Ethics approval and consent to participate}

Ethics was submitted to the Research Ethics Office at the University of Alberta and has been approved through independent review.

\section{Consent for publication}

Not applicable.

\section{Competing interests}

The authors declare that they have no competing interests.

\section{Publisher's Note}

Springer Nature remains neutral with regard to jurisdictional claims in published maps and institutional affiliations.

\section{Author details}

${ }^{1}$ Faculty of Medicine and Dentistry, University of Alberta, 8440-112 St. Edmonton, Edmonton, Alberta T6G 2B7, Canada. ${ }^{2}$ Department of Surgery, 2D4.27 Mackenzie Health Sciences Centre, University of Alberta, 8440-112 St. Edmonton, Edmonton, Alberta T6G 2B7, Canada.

Received: 2 May 2018 Accepted: 26 August 2018 Published online: 06 September 2018

\section{References}

1. Canadian Institute for Health Information. Health Care in Canada. A focus on seniors and aging. Ottawa. Ontario: CHH; 2011. p. 2011.

2. American College of Surgeons Committee on Trauma. ACS TQIP Geriatric Trauma Management Guidelines. https://www.facs.org/ /media/files/.../ trauma/tqip/geriatric\%20guide\%20taip.ashx. Accessed 1 Jan 2015.

3. Chu I, Vaca F, Stratton S, Chakravarthy B, Hoonpongsimanont W, Lotfipour S. Geriatric trauma care: challenges facing emergency medical services. Cal J Emerg Med. 2007;8:51-5.
4. Perdue PW, Watts DD, Kaufmann CR, Trask AL. Differences in mortality between elderly and younger adult trauma patients: geriatric status increases risk of delayed death. J Trauma-Injury Infec Crit Care. 1998;45:805-10.

5. Gowing R, Jain MK. Injury patterns and outcomes associated with elderly trauma victims in Kingston. Ontario Can J Surg. 2007;50:437-44.

6. Champion HR, Copes WS, Buyer D, Flanagan ME, Bain L, Sacco WJ. Major trauma in geriatric patients. Am J Public Health. 1989;79:1278-82.

7. Leading causes of death, Canada, 2008, males and females combined, counts (age-specific death rate per 100,000) [https://www.canada.ca/en/ public-health/services/reports-publications/leading-causes-deathhospitalization-canada/2008-males-females-combined-counts-specific-deathrate.html]. Oct 12th 2017

8. Brinkman-Stoppelenburg A, Rietjens JA, van der Heide A. The effects of advance care planning on end-of-life care: a systematic review. Palliat Med. 2014:28:1000-25.

9. Gordy S, Klein E. Advance directives in the trauma intensive care unit: do they really matter? Int J Crit IIIn Sci. 2011;1:132-7.

10. U.S. Department of Health and Human Services, U.S. Department of Health and Human Services Assistant Secretary for Planning and Evaluation Office of Disability Aging and Long-Term Care Policy, Advance Directive and Advance Care Planning. Washington, D.C: Report to Congress. https://aspe. hhs.gov/system/files/pdf/75811/ADCongRpt.pdf. Accessed 10 Oct 2017.

11. Fagerlin A, Schneider CE. Enough. The failure of the living will. Hast Cent Rep. 2004;34:30-42.

12. Perkins HS. Controlling death: the false promise of advance directives. Ann Intern Med. 2007;147:51-7.

13. Danis M, Southerland LI, Garrett JM, Smith JL, Hielema F, Pickard CG, Egner DM, Patrick DL. A prospective study of advance directives for life-sustaining care. N Engl J Med. 1991;324:882-8.

14. Detering $\mathrm{KM}$, Hancock $A D$, Reade MC, Silvester $W$. The impact of advance care planning on end of life care in elderly patients: randomised controlled trial. BMJ. 2010;23:c1345

15. Hammes BJ, Rooney BL, Gundrum JD. A comparative, retrospective, observational study of the prevalence, availability, and specificity of advance care plans in a county that implemented an advance care planning microsystem. J Am Geriatr Soc. 2010;58:1249-55.

16. Hoffman CB. Passion, persistence, and pennies. In: Rogne LM, Lauraine S, editors. Advance care planning: communicating about matters of life and death. New York: Springer Publishing Company, LLC; 2014.

17. Morrison RS, Morrison EW, Glickman DF. Physician reluctance to discuss advance directives. An empiric investigation of potential barriers. Arch Intern Med. 1994:154:2311-8

18. Winzelberg GS, Hanson LC, Tulsky JA. Beyond autonomy: diversifying endof-life decision-making approaches to serve patients and families. J Am Geriatr Soc. 2005;53:1046-50

19. Prendergast TJ. Advance care planning: pitfalls, progress promise. Crit Care Med. 2001;29:N34-9.

20. Teno J, Lynn J, Wenger N, Phillips RS, Murphy DP, Connors AF, Desbiens N, Fulkerson W, Bellamy P, Knaus WA. Advance directives for seriously ill hospitalized patients: effectiveness with the patient self-determination act and the SUPPORT intervention. SUPPORT investigators. Study to understand prognoses and preferences for outcomes and risks of treatment. J Am Geriatr Soc 1997:45:500-7.

Ready to submit your research? Choose BMC and benefit from:

- fast, convenient online submission

- thorough peer review by experienced researchers in your field

- rapid publication on acceptance

- support for research data, including large and complex data types

- gold Open Access which fosters wider collaboration and increased citations

- maximum visibility for your research: over $100 \mathrm{M}$ website views per year

At BMC, research is always in progress.

Learn more biomedcentral.com/submission 